\title{
Efeitos do Exercício Físico na Expressão e Atividade da AMPKa em Ratos Obesos Induzidos por Dieta Rica em Gordura
}

\author{
Effects of Physical Exercise in the Ampka Expression and Activity in \\ High-fat Diet Induced Obese Rats
}

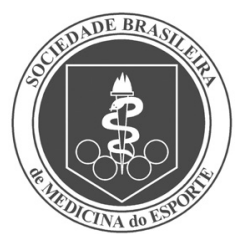

Artigo Original
José Rodrigo Pauli1,2,

Eduardo Rochete Ropelle',

Dennys Esper Cintra',

Cláudio Teodoro de Souza'

1. Departamento de Clínica Médica, FCM, Universidade Estadual de Campinas (Unicamp), Campinas, SP. Laboratório de investigação dos mecanismos moleculares de sinalização da insulina e dos efeitos moleculares do exercício físico no diabetes e obesidade 2. Departamento de Biociências, Faculdade de Educação Física - Modalidade Saúde, Universidade Federal de São Paulo, Campus Baixada Santista, Santos, SP.

\section{Endereço para correspondência: José Rodrigo Pauli. Rua XV de Novembro, 1.70113419235 \\ Piracicaba, SP. \\ E-mail: rodrigosere@yahoo.com.br}

Submetido em 23/11/2007

Versão final recebida em 30/06/2008 Aceito em 24/10/2008

\begin{abstract}
RESUMO
Introdução: A ingestão de dieta hiperlipídica é um fator de risco singular no desenvolvimento de resistência à insulina e diabetes do tipo 2. Objetivo: $O$ estudo investigou os efeitos do exercício físico na expressão e atividade da AMPKa em ratos obesos. Métodos: Foram utilizados ratos Wistar, aleatoriamente divididos em quatro grupos, que receberam dieta padrão de manutenção (grupo controle) ou dieta hiperlipídica (DHL) (grupos sedentários e exercitados), por período de quatro meses. Dois diferentes protocolos de exercícios foram utilizados: exercício agudo ou crônico de natação. O teste de tolerância à insulina foi realizado para estimar a sensibilidade à insulina. Os níveis protéicos da AMPKa e do GLUT4 e também de pAMPKa e pACC no músculo esquelético dos ratos foram determinados através da técnica de Western blot. Resultados: $O$ teste de tolerância à insulina revelou significativo prejuízo na ação da insulina após a alimentação com a DHL, indicando insulino-resistência quando comparado com grupo controle $(p<0,05)$. O tratamento por quatro meses com a DHL resultou em significativa redução no conteúdo protéico de AMPKa (2,2 vezes) e do GLUT4 (2,5 vezes) e nos níveis de p-AMPKa (2,4 vezes) e p-ACC (2,5 vezes) no músculo esquelético dos ratos sedentários quando comparado aos ratos controles. Ambos os protocolos de exercícios resultaram em aumento na fosforilação da AMPKa e ACC e aumento da sensibilidade à insulina, enquanto apenas o programa de exercício crônico promoveu o aumento da expressão dessas proteínas $(p<0,05)$. Conclusão: A alimentação com uma DHL causa redução na expressão e na atividade da AMPKa, enquanto a ativação da AMPKa pelo exercício físico melhora a sensibilidade à insulina, indicando que ratos obesos mantêm preservada a funcionalidade da via AMPKa.
\end{abstract}

Palavras-chave: obesidade, resistência à insulina, educação, treinamento físico.

\section{ABSTRACT}

Introduction: High-fat diet is a special risk factor in the development of insulin resistance and type 2 diabetes. Objective: To investigate the effects of physical exercise on the AMPK expression and activity in high-fat diet induced obese rats. Methods: Wistar rats were randomly divided into four groups and received either a rat maintenance diet (control group) or an isocaloric high-fat diet (HFD) (sedentary groups and exercised groups) for four months. Two different exercise protocols were utilized: acute or chronic swimming exercise. Insulin tolerance test was performed to estimate whole-body insulin sensitivity. AMPKa and GLUT4 as well as pAMPKa and pACC of rats' skeletal muscle levels were determined using Western blot. Results: Insulin tolerance test revealed a significantly impaired insulin action after HFDt feeding, indicating high-fat induced insulin resistance when compared to control group. Four months of HFD treatment induced to significant decrease of AMPKa (2.2-fold) and GLUT4 (2.5-fold) protein contents and also of p-AMPKa (2.4-fold) and p-ACC (2.5-fold) in sedentary rats' skeletal muscle when compared with the control group. Both exercise protocols resulted in increase of AMPKa and ACC phosphorylation and increase in insulin sensitivity, while chronic physical exercise alone provoked increase in these proteins expression $(p<0.05)$. Conclusion: High-fat feeding impairs AMPKa activity, while AMPKa activation by physical exercise improves insulin resistance, thus indicating that obese rats normally have the AMPK pathway preserved.

Keywords: obesity, insulin resistance, physical education and training.

\section{INTRODUÇÃO}

A ingestão de dieta hiperlipídica é um fator de risco singular no desenvolvimento de resistência à insulina e diabetes do tipo 2, causando aumento dos níveis plasmáticos de ácidos graxos livres e acúmulo excessivo de gordura em órgãos insulino-alvo ${ }^{(1,2)}$. Contudo, a relação entre dieta hiperlipídica e resistência à insulina é complexa, e o preciso mecanismo ainda não foi completamente elucidado. Uma das teorias mais aceitas até agora considera que a habilidade subnormal de órgãos-alvos à insulina em oxidar ácidos graxos seja um importante contribuinte da resistência à insulina induzida por exposição excessiva à gordura ${ }^{(3)}$. Estudos tanto in vivo ${ }^{(4,5)}$ como in vitro $^{(6)}$ têm demonstrado a relação entre oxidação intracelular de ácido graxo e 
sensibilidade à insulina. Por exemplo, foi demonstrado que a inibição da oxidação intracelular de ácidos graxos pode conduzir a um estado de resistência à insulina ${ }^{(4)}$, enquanto melhora na oxidação de ácido graxo no interior da célula causa maior resposta à insulina(6,7). Assim, é reconhecido que a dieta hiperlipídica induz quadro de resistência à insulina que é associada com prejuízo na capacidade de oxidação de ácidos graxos e aumento no acúmulo de gordura em tecidos-alvo à ação da insulina ${ }^{(8,9)}$; contudo, o mecanismo envolvido nessa condição é ainda pouco entendido.

Por outro lado, sabe-se que a contração muscular é eficiente em causar melhora na oxidação de gordura e aumentar a sensibilidade à insulina ${ }^{(3)}$. Nesse contexto, investigações em nível molecular têm trazido novos conhecimentos a respeito dos efeitos do exercício na captação de glicose. Dentre as inúmeras proteínas intracelulares estudadas, destaca-se a proteína quinase ativada por AMP (AMPK). A AMPK é uma molécula heterotrimérica que contém uma subunidade catalítica (a), com duas isoformas (a1 e a2), e duas subunidades regulatórias $(\beta$ e $\gamma)$, com as seguintes isoformas ( $\beta 1, \beta 2, \gamma 1$ y2 e $\gamma 3)$. Essa proteína é ativada pela fosforilação do resíduo de treonina 172 da alça de ativação da subunidade $a^{(10)}$. A ativação é causada pelo decréscimo no conteúdo energético celular. Na situação em que a relação AMP:ATP é aumentada, ocorre a mudança conformacional da molécula, deixando-a suscetível a fosforilação e ativação pela AMPK quinase (AMPKK) ${ }^{(11)}$. A AMPK fosforilada ativa vias que geram o aumento de ATP, tais como a oxidação de ácidos graxos, ao mesmo tempo em que inibe as vias anabólicas que consomem o ATP, tal como a síntese de ácidos graxos ( $A G)$.

Situações de estresse, como o exercício, hipóxia ou jejum prolongado podem ativar a AMPK através do aumento intracelular da razão AMP/ ATP. Na condição de exercício físico, a AMPK exerce papel importante na regulação do transporte de glicose durante a contração muscular ${ }^{(12,13)}$. A AMPK inativa a acetil CoA carboxilase (ACC) $)^{(12)}$, causando diminuição nos níveis intracelulares de Malonil CoA, um inibidor da carnitina acil transferase I (CAT I)(14). Portanto, o efeito da AMPK na oxidação de ácidos graxos é resultante da fosforilação e inibição da $A C C^{(15)}$.

Através da regulação da oxidação intracelular de ácidos graxos, a AMPK tem sido considerada uma proteína reguladora com efeito positivo na sensibilidade à insulina. Diversos agentes fisiológicos e farmacológicos exercem efeitos sensibilizantes à insulina via ativação da AMPK, como a metformina(16) , 5-aminoidazole-4-carboxamide-1- $\beta$ D-ribofuranoside (AICAR) ${ }^{(12)}$, exercício físico ${ }^{(17)}$, jejum prolongado ${ }^{(18)}$, etc. Recentemente, foi demonstrado que, em adição à oxidação de ácidos graxos, a AMPK pode regular a sensibilidade à insulina por estimular a expressão de GLUT4 ${ }^{(19)}$. O presente estudo teve por objetivo investigar os efeitos do exercício físico agudo e crônico na expressão e atividade da AMPKa e melhora da sensibilidade à insulina em ratos obesos induzidos por dieta rica em gordura.

\section{MÉTODOS}

Todos os experimentos foram conduzidos em acordo com os princípios e procedimentos de cuidado com o uso de animais experimentais e foram aprovados pelo comitê de ética da Universidade Estadual de Campinas. Os animais foram mantidos em ciclos de luz artificial de 12 horas claro e 12 horas escuro e alocados em gaiolas coletivas com quatro animais cada. Aleatoriamente, os ratos Wistar foram divididos em quatro grupos experimentais com similar peso corporal (250 \pm 5g): grupo controle (C), composto por ratos alimentados com ração padrão para roedores (ver detalhes da composição da dieta na tabela 1); grupo obeso sedentário (OB-S), composto por ratos que receberam dieta hiperlipídica por período de quatro meses (ver detalhes da dieta na tabela 1); grupo obeso exercitado (OB-E), composto por animais que receberam a dieta rica em lípides por quatro meses e realizaram uma sessão única de exercício de natação; grupo obeso treinado (OB-T), composto por animais que receberam dieta hiperlipídica por quatro meses e foram submetidos a um programa crônico de exercício físico de natação.

Tabela 1. Composição da dieta padrão e da dieta rica em gordura.

\begin{tabular}{l|c|c|c|c}
\hline \multicolumn{1}{c|}{ Ingredientes } & $\begin{array}{c}\text { Ração padrão } \\
\mathbf{( g / k g )}\end{array}$ & Kcal/kg & $\begin{array}{c}\text { Ração } \\
\text { hiperlipídica } \\
\mathbf{( g / k g )}\end{array}$ & Kcal/kg \\
\hline Amido de milho (q.s.p) & 397,5 & 1.590 & 115,5 & 462 \\
\hline Caseina & 200 & 800 & 200 & 800 \\
\hline Sacarose & 100 & 400 & 100 & 400 \\
\hline Amido dextrinado & 132 & 528 & 132 & 528 \\
\hline Banha de porco & - & - & 312 & 2.808 \\
\hline Oleo de soja & 70 & 630 & 40 & 360 \\
\hline Celulose & 50 & - & 50 & - \\
\hline Mistura de minerais & 35 & - & 35 & - \\
\hline Mistura de vitaminas & 10 & - & 10 & - \\
\hline -cistina & 3 & - & 3 & - \\
\hline Colina & 2,5 & - & 2,5 & - \\
\hline Total & 1.000 & $\mathbf{3 . 9 4 8}$ & $\mathbf{1 . 0 0 0}$ & $\mathbf{5 . 3 5 8}$ \\
\hline
\end{tabular}

\section{Programa de exercício físico agudo}

Para adaptação ao exercício de natação, os ratos foram primariamente inseridos ao meio liquido durante 10 minutos por dois dias consecutivos. Os ratos obesos (OB-E) nadaram em grupos de quatro em baldes plásticos de $45 \mathrm{~cm}$ de diâmetro e com $60 \mathrm{~cm}$ de profundidade, por duas sessões de três horas, separadas por intervalo de 45 minutos; a temperatura da água foi mantida em aproximadamente $34^{\circ} \mathrm{C}$. O protocolo de exercício agudo foi anteriormente utilizado em outros estudos de nosso laboratório(1,20)

\section{Programa de treinamento físico crônico}

O treinamento consistiu de natação, em grupos de quatro animais, nas mesmas condições em que os ratos realizaram a sessão única de exercício. Os ratos do grupo OB-T nadaram uma hora por dia, cinco dias por semana, durante oito semanas, suportando uma carga que foi progressivamente aumentada de 2,5\% a 5\% do peso corporal, ajustada semanalmente, de acordo com o peso do animal na semana. Esse protocolo tem sido previamente utilizado em nosso laboratório(21).

\section{Avaliações de parâmetros fisiológicos e metabólicos}

Inicialmente, foram realizadas avaliações de alguns parâmetros metabólicos nos ratos. Para isso, os animais permaneceram em repouso por 36 horas após a última sessão de exercício (aguda ou crônica). Os ratos tiveram acesso livre à água e ração; no entanto, ficaram sob abstinência de alimento por um período de seis horas.

A massa corporal dos animais foi determinada em balança analítica. Amostras de sangue foram coletadas da veia porta dos ratos através de seringa de $1 \mathrm{ml}$ e posteriormente centrifugadas a 3.500rpm por 15 minutos. Imediatamente após centrifugação, o soro foi armazenado 
em freezer a $-80^{\circ} \mathrm{C}$ para posterior dosagem de glicose e insulina. A concentração plasmática de glicose foi avaliada através do método oxidase enzimático calorimétrico e a concentração de insulina plasmática pelo método de radioimunoensaio.

\section{Teste de tolerância à insulina intraperitoneal (TTlip)}

Foi respeitado um intervalo de uma hora tanto para os animais submetidos ao exercício agudo como também aos do protocolo de exercício crônico, para que fossem realizados os procedimentos experimentais. Nesse período de uma hora os animais permaneceram sob abstinência de água e ração. Adverte-se que apenas os ratos utilizados no TTlip receberam insulina intraperitoneal, como descrito a seguir.

Através de corte na extremidade da cauda do animal, foi realizada uma primeira coleta de sangue para a dosagem de glicose, o que equivale ao tempo zero (t0) do teste. Após isso, a insulina (2U/kg de peso corporal) foi injetada intraperitonealmente e amostras de sangue foram coletadas através da extremidade da cauda dos animais nos tempos 5, 10, 15, 20, 25 e 30 minutos para a determinação da glicose, como descrito em estudos prévios em nosso laboratório ${ }^{(1,20)}$. A velocidade constante do decaimento da glicose (Kitt) foi calculada usando a formula 0,693/t1/2. O t1/2 da glicose foi calculado a partir da curva da análise dos mínimos quadrados da concentração da glicose durante a fase de decaimento linear ${ }^{(22)}$.

\section{Sacrifício e extração do músculo esquelético}

Imediatamente após uma hora da ultima sessão de exercício e sob abstinência de água e ração, os ratos foram anestesiados através da administração intraperitoneal de tiopental sódico $(40 \mathrm{mg} / \mathrm{kg}$ ) e a perda dos reflexos pedal e corneano foi usada como controle da anestesia. Fatias do músculo gastrocnêmio foram retiradas e homogeneizadas em tampão de imunoprecipitação contendo 1\% de Triton X 100, 100mM de Tris (pH 7,4), 100mM de pirofosfato de sódio, $100 \mathrm{mM}$ de fluoreto de sódio, 10mM de EDTA, 10mM de vanadato de sódio, 2mM de PMSF e $0,1 \mathrm{mg} / \mathrm{mL}$ de aprotinina a $4^{\circ} \mathrm{C}$. O homogeneizado foi então centrifugado a 11.000rpm por 30 minutos. No sobrenadante, foi determinada a concentração de proteínas utilizando o método de Bradford(23) e posteriormente realizada a determinação do extrato total. As análises de proteínas intracelulares pela técnica de Western blot estão descritas a seguir.

\section{Western blot}

O extrato total foi ressuspenso em tampão de Laemmli, contendo $100 \mathrm{mmol} / \mathrm{L}$ de DTT ${ }^{(24)}$. Após rápida fervura, foram aplicados em gel de poliacrilamida para separação por eletroforese (SDS-PAGE). As proteínas separadas em SDS-PAGE foram transferidas para membrana de nitrocelulose em aparelho de transferência da BIO-RAD, como previamente descrito ${ }^{(25)}$. A membrana de nitrocelulose foi incubada overnight com anticorpo especifico. Os anticorpos utilizados foram anti-AMPKa, anti-ACC, antiphospho-AMPKa, antiphospho-ACC provenientes da Cell signaling Technology (Beverly, MA, EUA), e anti-GLUT4 proveniente da Santa Cruz Biotechnology (Santa Cruz, CA, EUA). A ligação de anticorpo a proteínas não-específicas foi minimizada pela pré-incubação da membrana de nitrocelulose com tampão de bloqueio (5\% de leite em pó desnatado; $10 \mathrm{mmol} / \mathrm{L}$ de Tris; $150 \mathrm{mmol} / \mathrm{L}$ de $\mathrm{NaCl} ; 0,02 \%$ de Tween 20) por duas horas. $\mathrm{O}$ sinal foi detectado por tratamento com $2 \mathrm{mCi}$ de [125l] Proteína A (30mCi/mg) em 10mL de tampão de bloqueio por duas horas em temperatura ambiente e exposição a filmes de RX Kodak a $-80^{\circ} \mathrm{C}$ de $12-48$ horas. As bandas identificadas na auto-radiografia foram quantificadas através de densitometria óptica pelo programa Scion Image software (ScionCorp, Frederick, MD).

\section{ANÁLISE ESTATÍSTICA}

Os resultados estão expressos em média \pm erro padrão da média. Quando comparados dois grupos, foi utilizado o teste $t$ de Student para dados não pareados. Quando necessário, foi utilizada análise de variância seguida de teste de comparação múltipla de médias. Em todos os procedimentos estatísticos, o nível de significância foi prefixado em $P<0,05$.

\section{RESULTADOS}

\section{Parâmetros fisiológicos e metabólicos}

Massa corporal, níveis de glicose e de insulina plasmática foram mensurados e os dados encontram-se sumarizados na tabela 2. Ratos alimentados com a dieta hiperlipídica tiveram aumento significativo da massa corporal (OB-S, 610,8 \pm 14,4g; OB-E, 614,1 \pm 21,6g; OB-T, 595,1 1 $23,5 \mathrm{~g})$ em relação aos ratos controles $(436,3 \pm 14,4 \mathrm{~g})$. Com relação à insulina, não foram encontradas diferenças significativas entre animais controles e obesos. Já a concentração de insulina sérica foi maior nos animais que receberam a dieta hiperlipídica (OB-S, 7,12 \pm 0,86; OB-E, $\left.6,96 \pm 1,2 \mathrm{ng} / \mathrm{ml}^{-1}\right)$, com exceção dos submetidos ao exercício crônico (OB-T, 3,29 $\left.\pm 1,1 \mathrm{ng} / \mathrm{ml}^{-1}\right)$, que apresentaram valores semelhantes aos dos ratos controles $\left(2,89 \pm 0,8 \mathrm{ng} / \mathrm{ml}^{-1}\right)$. Por fim, verifica-se que ratos sedentários alimentados com a dieta rica em gordura tiveram menor taxa de consumo de glicose quando comparados com os controles $(5,4 \pm 0,4 ;$ OB-S, $1,8 \pm 0,6 \% / \mathrm{min})$, respectivamente. Por outro lado, tanto os animais que realizaram o exercício agudo quanto o crônico tiveram aumento na taxa de captação de glicose no TTlip (OB-E, 4,36 $\pm 0,8 ;$ OB-T, $5,1 \pm 1,0 \% / \mathrm{min})$.

Tabela 2. Valores médios e erro padrão da média dos parâmetros fisiológicos e metabólicos dos animais

\begin{tabular}{|c|c|c|c|c|}
\hline & Controle & OB-S & OB-E & OB-T \\
\hline $\begin{array}{l}\text { Peso corporal (g) } \\
(n=8)\end{array}$ & $436,3 \pm 14,4$ & $610,8 \pm 25,8^{*}$ & $614,1 \pm 21,6^{*}$ & $595,1 \pm 23,5^{*}$ \\
\hline $\begin{array}{l}\text { Glicose plasmática }\left(\mathrm{mg} / \mathrm{dl}^{-1}\right) \\
(\mathrm{n}=8)\end{array}$ & $79,86 \pm 3,9$ & $94,90 \pm 8,1$ & $92,66 \pm 6,3$ & $86,12 \pm 5,6$ \\
\hline $\begin{array}{l}\text { Insulina }\left(\mathrm{ng} / \mathrm{ml}^{-1}\right) \\
(\mathrm{n}=8)\end{array}$ & $2,89 \pm 0,8$ & $7,12 \pm 0,86^{*}$ & $6,96 \pm 1,2^{*}$ & $3,29 \pm 1,1^{\#}$ \\
\hline $\begin{array}{l}\text { TTI }\left(\% / \min ^{-1}\right) \\
(n=8)\end{array}$ & $5,4 \pm 0,4$ & $1,8 \pm 0,6^{* \#}$ & $4,36 \pm 0,8$ & $5,1 \pm 1,0$ \\
\hline
\end{tabular}

${ }^{*} \mathrm{P}<0,05$, vs ratos controles $\mathrm{e}^{\# \mathrm{P}}<0,05$, vs ratos OB-S e OB-E.

\section{Expressão e fosforilação da AMPKa e da ACC}

Imediatamente após uma hora da última sessão de exercício houve redução nos níveis protéicos de AMPKa no músculo gastrocnêmio dos animais sedentários alimentados com a dieta rica em gordura em 2,2 vezes se comparado com os ratos controles, indicando que esse tipo de dieta prejudica a expressão de AMPKa. Por outro lado, o treinamento de natação por oito semanas aumentou em aproximadamente 2,0 e 1,5 vezes a expressão de AMPKa se comparado com o dos ratos OB-S e OB-T, respectivamente (figura $1 \mathrm{~A}$ ).

A atividade da AMPKa no músculo gastrocnêmio dos animais foi avaliada através da mensuração dos níveis de AMPK fosforilada ( $p$ AMPKa) (figura 1B). Comparados com os controles, ratos alimentados com dieta rica em lípides tiveram redução dos níveis de p-AMPKa em 2,4 vezes, indicando que este tipo de dieta pode também prejudicar a atividade da AMPKa. Por outro lado, observa-se que os ratos submetidos tanto ao exercício crônico quanto ao agudo tiveram aumento 
na fosforilação da AMPKa em aproximadamente 2,0 e 1,9 vezes, se comparados com os ratos controles, e aumento de 4,5 e 4,6 vezes em relação aos do grupo OB-S, respectivamente. Tal fato demonstra a eficácia do exercício na ativação da AMPKa.

Por fim, o efeito biológico da AMPK é regular a oxidação de ácidos graxos através da fosforilação e inativação da $A C C^{(14)}$. Verifica-se que os animais expostos à dieta hiperlipídica tiveram redução dos níveis de ACC fosforilada em 2,5 vezes quando comparados com os do grupo controle. Os animais exercitados, independente do protocolo (agudo ou crônico), apresentaram aumento da pACC em aproximadamente 1,8 e 1,9 vezes em relação aos ratos controles. Quando comparados com os ratos do grupo OB-S, os ratos submetidos ao protocolo de exercício agudo e crônico tiveram aumento da fosforilação da ACC de 4,6 e 4,8 vezes, respectivamente. Portanto, esses dados não só sugerem que a dieta rica em lípides afeta a expressão e a atividade da AMPKa, mas também indica que a insuficiência na oxidação de ácidos graxos mediada pela ACC pode ser um mecanismo efetor importante da resistência à insulina nesse modelo experimental.

\section{Expressão de GLUT4}

Na figura 2, observa-se que os animais sedentários expostos à dieta rica em gordura tiveram redução significativa na expressão do GLUT4 no músculo esquelético de 2,5 vezes quando comparado com os ratos controles. Porém, observa-se que o exercício crônico aumentou a expressão de GLUT4 em aproximadamente 3,1 e 2,4 vezes quando comparada com a dos animais OB-S e OB-E, respectivamente. Esses resultados indicam que o mecanismo mediado por AMPK/GLUT4 pode participar da resistência à insulina induzida por dieta rica em gordura.

\section{DISCUSSÃO}

Diversos estudos demonstraram que a ingestão de dieta rica em lípides está associada com resistência à insulina em humanos(26) e em animais $^{(1,20)}$. Embora, tanto ácidos graxos insaturados como também os saturados poderem causar resistência à insulina, existem evidências de que a ingestão de gordura saturada é mais efetiva em induzir alterações na ação da insulina ${ }^{(26,27)}$. A banha de porco constitui-se em um tipo de ácido graxo saturado amplamente consumido por humanos e por isso foi escolhida no presente estudo.

Estudos prévios realizados em nosso laboratório mostraram que animais submetidos a uma dieta rica em lípides por quatro ou 16 sema-

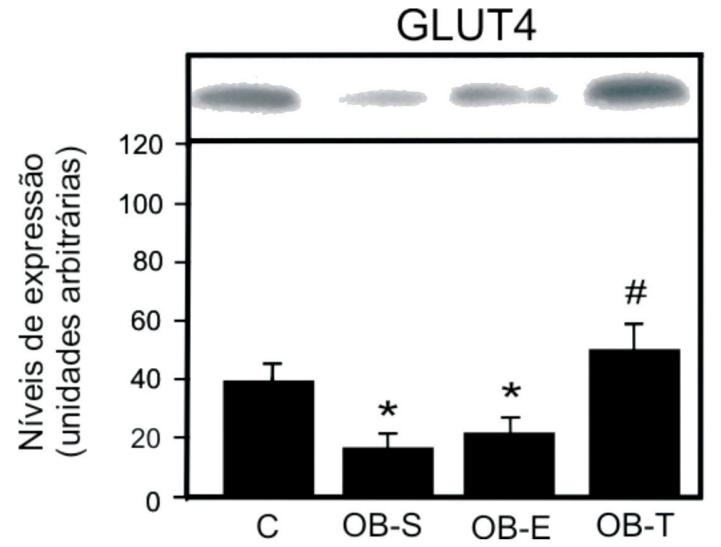

Figura 2. Expressão do GLUT4 no músculo gastrocnêmio dos ratos controles, OB-S, OB-E e OB-T. Barras representam média \pm erro padrão da média de oito animais por grupo. ${ }^{*} P<0,05$, vs ratos controles e $\# P<0,05$, vs ratos OB-S e OB-E.

nas tiveram alteração na taxa de decaimento da glicose durante o teste de tolerância à insulina intraperitoneal $(\mathrm{TTI})^{(1,20,28)}$. No presente estudo, observa-se que ratos submetidos a uma dieta hiperlipídica (DHL) por período de quatro meses tiveram redução significativa na percentagem de glicose captada durante o TTI, quando comparados com os animais alimentados com uma dieta padrão. Além disso, verifica-se maior concentração de insulina sérica nos animais alimentados com a DHL, com exceção do grupo OB-T, em comparação com os animais controles. Portanto, tais resultados demonstram que a DHL utilizada em nosso estudo foi eficaz em induzir resistência à insulina.

Como proposto por McGarry ${ }^{(25)}$, a ineficiência da oxidação intracelular de ácido graxo e o acúmulo dos depósitos de gordura no interior de órgãos-alvo à insulina são um dos possíveis mecanismos de resistência à insulina induzida por dieta rica em lípides Pesquisadores têm investigado a relação entre oxidação intracelular de ácidos graxos e sensibilidade à insulina ${ }^{(4,6)}$. Embora os efeitos da exposição à dieta rica em lípides sobre distúrbios no metabolismo intracelular de ácidos graxos venha sendo bem demonstrada em humanos ${ }^{(30,31)}$ e roedores $^{(8,32)}$, o preciso mecanismo ainda não é totalmente conhecido. A AMPK desempenha papel- chave na $\beta$-oxidação de ácidos graxos e

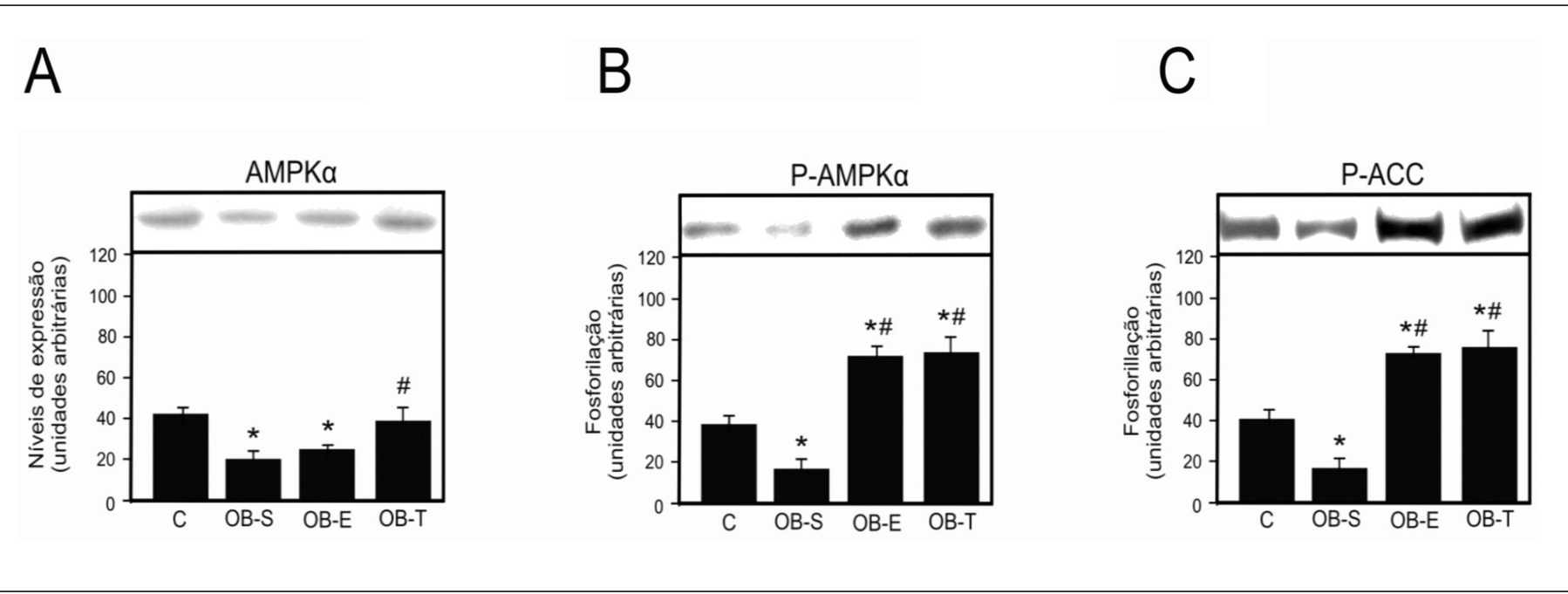

Figura 1. Expressão e fosforilação da AMPKa no músculo gastrocnêmio (A e B) e fosforilação da ACC (C) dos ratos controle, OB-S, OB-E e OB-T. Barras representam média \pm erro padrão da média de oito animais por grupo. ${ }^{*} \mathrm{P}<0,05$, vs ratos controles $\mathrm{P}^{\#} \mathrm{P}<0,05$, vs ratos OB-S e OB-E. 
regulação da sensibilidade à insulina. No entanto, seu efeito contra a lipotoxicidade ainda é incerto. Neste estudo, investigamos o possível papel da AMPKa na resistência à insulina em músculo esquelético de ratos obesos induzida por ingestão de dieta hiperlipídica.

A AMPK é composta por três subunidades, $a, \beta$ e $\gamma$. Dentre essas, a subunidade alfa (AMPKa) é a subunidade catalítica da proteína. Para observar os efeitos da exposição à dieta hiperlipídica na AMPK, os níveis protéicos da AMPKa em músculo esquelético de ratos foram mensurados usando a técnica de Western blot. Os resultados mostram que quatro meses de alimentação com a dieta hiperlipídica foram suficientes para causar redução no conteúdo da proteína AMPKa nos ratos. Além disso, quando se avaliou a atividade dessa enzima através da mensuração da fosforilação da subunidade alfa (p-AMPKa), a alimentação com a dieta hiperlipídica também resultou em níveis menores de fosforilação da AMPKa no músculo esquelético dos ratos, sugerindo prejuízo na atividade da AMPKa pela exposição à dieta hiperlipídica. Desse modo, nossos resultados fornecem evidências de que a dieta rica em gordura saturada induz resistência à insulina e é acompanhada por prejuízo na expressão e atividade da AMPKa, indicando um possível papel importante dessa proteína na lipotoxicidade.

O maior efeito biológico da AMPK na regulação da oxidação intracelular de ácidos graxos se dá através da fosforilação e inativação da ACC $^{(14)}$. Como demonstrado no presente trabalho, similarmente à redução da expressão e atividade da AMPKa, os níveis de PACC diminuíram nos animais que receberam a dieta hiperlipídica. A redução nos níveis de ACC fosforilada leva ao aumento da atividade da ACC, aumento do conteúdo de malonil-CoA, prejuízo na oxidação de ácidos graxos e, consequentemente, resistência à insulina. Portanto, a ACC pode atuar como um efetor distal à via de sinalização da AMPK no possível mecanismo de resistência à insulina induzida por dieta hiperlipídica. O aumento na atividade da ACC pode conduzir ao início da resistência à insulina, enquanto a inativação desta tem efeito sensibilizante à insulina ${ }^{(5,15)}$.

Além disso, existem evidências de que a AMPK aumenta a eficiência mitocondrial em oxidar ácidos graxos e, consequentemente, aumenta a sensibilidade à insulina. O tratamento crônico com AICAR, um ativador farmacológico dessa proteína, promove adaptações similares às promovidas pelo exercício, como aumento do conteúdo de citocromo $\mathrm{C}$ e das enzimas mitocondriais citrato sintase, succinato desidrogenase e malato desidrogenase ${ }^{(33)}$. O efeito molecular semeIhante entre os ativadores farmacológicos da AMPK e do treinamento físico não é totalmente conhecido; no entanto, alguns estudos sugerem que isso envolve o aumento da expressão do PGC1-a (peroxisome proliferator-activated receptor-gamma coactivatorlalpha), um mediador de biogênese mitocondrial. Embora, no presente estudo, não tenha sido avaliada a expressão do PGC1-a no músculo esquelético, sabe-se que ratos submetidos a treinamento de natação apresentam maior expressão gênica do PGC1-a no músculo e que esse efeito é reproduzido pela incubação de músculo isolado em solução contendo $\operatorname{AICAR}^{(34)}$.

Em nosso estudo, verificamos que a ativação da AMPK através dos protocolos de exercício agudo ou crônico facilita o transporte de glicose e melhora a resistência à insulina no músculo esquelético de ratos obesos induzidos por dieta hiperlipídica. Em acordo com nossos resultados, pesquisadores demonstraram recentemente que indivíduos diabéticos do tipo 2, com índice de massa corporal entre 26 e 29kg/m², têm aumento da atividade da AMPK através do uso de drogas ou pelo exercício físico ${ }^{(35,36,37)}$. Contudo, o mesmo não acontece em condição de severa resistência à insulina, em que ratos obesos Zucker apresentam redução significativa na via de sinalização da $\mathrm{AMPK}^{(38)}$. Portanto, não se descarta a possibilidade de os animais, uma vez permanecendo sob alimentação rica em gordura por um período superior a quatro meses, poderem apresentar alterações mais severas sobre a funcionalidade da via AMPK e, consequentemente, na sinalização da insulina.

Além do efeito na sensibilidade à insulina via regulação da oxidação de ácidos graxos, a AMPK pode também regular a expressão de GLUT4. Como demonstrado por Jessen et al. ${ }^{(19)}$, uma única injeção de AICAR resultou em aumento de 78\% da atividade da AMPK e de 97,8\% do conteúdo protéico de GLUT4 no músculo esquelético de ratos. O GLUT4 tem papel essencial no mecanismo de sinalização celular da insulina e sua expressão também é diminuída pela ingestão de dieta hiperlipídica, como demonstrado no presente estudo. Portanto, apesar de a possibilidade de outros mecanismos estarem envolvidos na diminuição da expressão do GLUT4, o decréscimo na expressão e atividade da AMPK pode ter contribuído, no mínimo em parte, para essa redução nos animais obesos induzidos por dieta hiperlipídica. Assim, o GLUT4 pode também atuar como outro efetor importante no possível mecanismo mediado pela AMPK de resistência à insulina induzida por dieta hiperlipídica. MEF2 e GEF são dois reguladores de transcrição do GLUT4 e, de acordo com algumas pesquisas, a AMPK aumenta a expressão e atividade deles ${ }^{(39,40)}$. Acredita-se que esse seja o possível mecanismo pela qual a AMPK tenha efeito regulatório na transcrição de GLUT4.

Todavia, parece claro que apenas o programa de exercício regular de natação é capaz de promover aumento na fosforilação da AMPKa e ACC, e também aumento na expressão de AMPKa e GLUT4, fato esse não detectado nos animais submetidos apenas à sessão aguda de exercício. Além disso, deve-se destacar que o treinamento físico também foi favorável para a redução dos níveis plasmáticos de insulina, como verificado nos animais OB-T, que apresentaram valores desse hormônio semelhantes aos dos ratos controles. A concentração maior de insulina sérica evidenciada nos animais OB-E em relação aos animais OB-T em condições basais (36 horas após a última sessão de esforço físico) reforça a necessidade do treinamento sistematizado para evitar a hiperinsulinemia em ratos obesos insulino-resistentes induzida por dieta hiperlipídica, e manutenção da homeostase glicêmica.

É possível que outras adaptações decorrentes do treinamento físico, como aumento na densidade mitocondrial, na vascularização periférica, de hemodinâmica, entre outras, favoreçam a entrada de glicose e oxidação de gordura, o que explicaria, no mínimo em parte, os valores menores de insulina nos ratos OB-T em comparação com os ratos OB-E. No entanto, sabe-se que a atividade da AMPK se dá por algumas horas após o exercício e, por isso, a melhora na expressão de GLUT4 no músculo esquelético dos ratos obesos treinados parece ser uma adaptação determinante para a captação de glicose. Semelhante ao nosso estudo, Luciano et al. (21) demonstraram que o exercício crônico aumenta a expressão de GLUT4 e isso resulta em melhora no metabolismo da glicose. Por conseguinte, apesar do efeito favorável do exercício agudo na captação de glicose, sugere-se que é fundamental se manter fisicamente ativo para obtenção de resultados duradouros e preventivos sobre a resistência à insulina induzida pela ingestão de dieta hiperlipídica. Embora o conhecimento a respeito dos efeitos moleculares do exercício físico, principalmente do treinamento sobre a captação de glicose e oxidação de ácidos graxos não seja totalmente compreendido, pode-se dizer através dos resultados deste estudo que a AMPK tem papel intrigante na homeostase da glicose, participando tanto na translocação e também na transcrição do GLUT4 e, ainda, melhorando a oxidação de ácidos graxos através da ativação da ACC. Embora, possivelmente, existam outros mecanismos intracelulares importantes para a homeostase da glicose, como pre- 
viamente apresentados, e que não foram avaliados em nosso estudo, sugere-se que tanto o aumento da expressão de GLUT4 e também da ACC fosforilada sejam mecanismos importantes na melhora da ação da insulina.

Em síntese, nossos resultados mostram que houve redução na expressão e atividade da AMPKa nos animais que receberam a dieta hiperlipídica, e isso foi acompanhado por alteração nos níveis de ACC, do GLUT4 e de resistência à insulina. Por outro lado, o exercício físico agudo ou crônico melhorou a sensibilidade à insulina e aumentou a captação de glicose. No entanto, apesar do aumento da atividade da AMPKa e da ACC com ambos os protocolos de exercícios (agudo e crônico), somente o treinamento de natação foi capaz de induzir mudanças na expressão da AMPKa e do GLUT4 no músculo esquelético e, ainda, manter os níveis insulinêmicos semelhantes aos dos ratos controles, o que demonstra que o programa de treinamento sistematizado tem maior eficácia sobre as anormalidades moleculares associadas à obesidade e resistência à insulina em ratos expostos em longo prazo à dieta rica em gordura.

Todos os autores declararam não haver qualquer potencial conflito de interesses referente a este artigo.

\section{REFERÊNCIAS BIBLIOGRÁFICAS}

1. Pauli JR, Ropelle ER, Cintra DE, Carvalho-Filho MA, Moraes JC, De Souza CT, et al. Acute physical exercise reverses S-nitrosation of the insulin receptor, insulin receptor substrate 1 and protein kinaseB/Akt in diet-induced obese Wistar rats. J Physiol. 2008;586(2):659-71.

2. Cintra DE, Pauli JR, Araújo EP, Moraes JC, de Souza CT, Milanski M, et al. Interleukin-10 is a protective factor against diet-induced insulin resistance in liver. J Hepatol. 2008;48(4):628-37.

3. Thyfault JP, Cree MG, Zheng D, Zwetsloot JJ, Tapscott EB, Koves TR, et al. Contraction of insulin-resistant muscle normalizes insulin action in association with increased mitochondrial activity and fatty acid catabolism. Am J Physiol Cell Physiol. 2007;292(2):C729-39.

4. Dobbins RL, Szczepaniak LS, Bentley B, Esser V, Myhill J, McGarry JD. Prolonged inhibition of muscle carnitine palmitoyltransferase-1 promotes intramyocellular lipid accumulation and insulin resistance in rats. Diabetes. 2001;50(1):123-30.

5. Abu-Elheiga L, Oh W, Kordari P, Wakil SJ. Acetyl-CoA carboxylase 2 mutant mice are protected against obesity and diabetes induced by high-fat/high-carbohydrate diets. Proc Natl Acad Sci U S A. 2003;100(18):10207-12

6. Perdomo G, Commerford SR, Richard AM, Adams SH, Corkey BE, O'Doherty RM, et al. Increased beta-oxidation in muscle cells enhances insulin-stimulated glucose metabolism and protects against fatty acid-induced insulin resistance despite intramyocellular lipid accumulation. J Biol Chem. 2004;279(26):27177-86.

7. OhW, Abu-Elheiga L, Kordari P, Gu Z, ShaikenovT, Chirala SS, et al. Glucose and fat metabolism in adipose tissue of acetyl-CoA carboxylase-2 knockout mice. Proc Natl Acad Sci U S A. 2005;102(5):1384-9.

8. Kim CH, Kim MS, Youn JY, Park HS, Song HS, Song KH, et al. Lipolysis in skeletal muscle is decreased in high-fat-fed rats. Metabolism. 2003;52(12):1586-92.

9. Storlien LH, Jenkins AB, Chisholm DJ, Pascoe WS, Khouri S, Kreagen EW. Influence of dietary fat composition on development of insulin resistance in rats. Relationship to muscle triglyceride and and omega-3 fatty acids in muscle phospholipid. Diabetes. 1991;40(2):280-9.

10. Hardie DG, Carling D. The AMP-activated protein kinase - fuel gauge of the mammalian cell? Eur J Biochem. 1997;246(2):259-73. Review.

11. Hardie DG, Scott JW, Pan DA, Hudson ER. Management of cellular energy by the AMP-activated protein kinase system. FEBS Lett. 2003;546(1):113-20. Review.

12. Ai H, Ihlemann J, Hellsten Y, Lauritzen HP, Hardie DG, Galbo H, et al. Effect of fiber type and nutritiona state on AICAR- and contraction-stimulated glucose transport in rat muscle. Am J Physiol Endocrinol Metab. 2002;282(6):E1291-300.

13. Vavvas D, Apazidis A, Saha AK, Gamble J, Patel A, Kemp BE, et al. Contraction-induced changes in acetyl-CoA carboxylase and 5'-AMP-activated kinase in skeletal muscle. J Biol Chem. 1997;272(20):13255-61.

14. Rutter GA, da Silva Xavier G, Leclerc I. Roles of 5'-AMP-activated protein kinase (AMPK) in mammalian glucose homeostasis. Biochem J. 2003;375(Pt 1):1-16. Review.

15. Savage DB, Choi CS, SamueIVT, Liu ZX, Zhang D, Wang A, et al. Reversal of diet-induced hepatic steatosis and hepatic insulin resistance by antisense oligonucleotide inhibitors of acetyl-CoA carboxylases 1 and 2. J Clin Invest. 2006;116(3):817-24

16. Zhou G, Myers R, Li Y, Chen Y, Shen X, Fenyk-Melody J, et al. Role of AMP-activated protein kinase in mechanism of metformin action. J Clin Invest. 2001;108(8):1167-74.

17. Musi N, Fujii N, Hirshman MF, Ekberg I, Fröberg O, Ljungqvist O, et al. AMP-activated protein kinase (AMPK) is activated in muscle of subjects with type 2 diabetes during exercise. Diabetes. 2001;50(5):921-7.

18. De Lange P, Moreno M, Silvestri E, Lombardi A, Goglia F, Lanni A. Fuel economy in food-deprived skeletal muscle: signaling pathways and regulatory mechanisms. FASEB J. 2007;21(13):3431-41. Review.

19. Jessen N, Pold R, Buhl ES, Jensen LS, Schmitz O, Lund S. Effects of AICAR and exercise on insulin-stimulated glucose uptake, signaling, and GLUT-4 content in rat muscles. J Appl Physiol. 2003;94(4):1373-9.

20. Ropelle ER, Pauli JR, Prada PO, de Souza CT, Picardi PK, Faria MC, et al. Reversal of diet-induced insulin resistance with a single bout of exercise in the rat: the role of PTP1B and IRS-1 serine phosphorylation. J Physiol. 2006;577(Pt 3):997-1007.
21. Luciano E, Carneiro EM, Carvalho CR, Carvalheira JB, Peres SB, Reis MA, et al. Endurance training improves responsiveness to insulin and modulates insulin signal transduction through the phosphatidylinositol 3- kinase/Akt-1 pathway. Eur J Endocrinol. 2002;147(1):149-57.

22. Bonora E, Moghetti P, Zancanaro C, Cigolini M, Querena M, Cacciatori V, et al. Estimates of in vivo insulin action in man: comparison of insulin tolerance tests with euglycemic and hyperglycemic glucose clamp studies. J Clin Endocrinol Metab. 1989;68(2):374-8

23. Bradford MM. A rapid and sensitive method for the quantitation of microgram quantities of protein utilizing the principle of protein-dye binding. Anal Biochem. 1976;72:248-54.

24. Laemmli UK. Cleavage of structural proteins during the assembly of the head of bacteriophage T4. Nature. 1970;227(5259):680-5.

25. Saad MJ, Maeda L, Brenelli SL, Carvalho CR, Paiva RS, Velloso LA. Defects in insulin signal transduction in liver and muscle of pregnant rats. Diabetologia. 1997;40(2):179-86.

26. Boden G. Role of fatty acids in the pathogenesis of insulin resistance and NIDDM. Diabetes. 1997:46(1):3-10. Review.

27. Hunnicutt JW, Hardy RW, Williford J, McDonald JM. Saturated fatty acid-induced insulin resistance in rat adipocytes. Diabetes. 1994;43(4):540-5.

28. Prada PO, Pauli JR, Ropelle ER, Zecchin HG, Carvalheira JB, Velloso LA, et al. Selective modulation of the CAP/Cbl pathway in the adipose tissue of high fat diet treated rats. FEBS Lett. 2006;580(20):4889-94.

29. McGarry JD. Banting lecture 2001: dysregulation of fatty acid metabolism in the etiology of type 2 diabetes. Diabetes. 2002;51(1):7-18.

30. Astrup A, Buemann B, Christensen NJ, Toubro S. Failure to increase lipid oxidation in response to increasing dietary fat content in formerly obese women. Am J Physiol. 1994;266(4 Pt 1):E592-9.

31. Phillips DI, Caddy S, Ilic V, Fielding BA, Frayn KN, Borthwick AC, et al. Intramuscular triglyceride and muscle insulin sensitivity: evidence for a relationship in nondiabetic subjects. Metabolism. 1996:45(8):947-50

32. Oakes ND, Cooney GJ, Camilleri S, Chisholm DJ, Kraegen EW. Mechanisms of liver and muscle insulin resistance induced by chronic high-fat feeding. Diabetes. 1997;46(11):1768-74.

33. Winder WW, Holmes BF, Rubink DS, Jensen EB, Chen M, Holloszy JO. Activation of AMP-activated protein kinase increases mitochondrial enzymes in skeletal muscle. J Appl Physiol. 2000;88(6):2219-26.

34. Terada S, Goto M, Kato M, Kawanaka K, Shimokawa T, Tabata I. Effects of low-intensity prolonged exercise on PGC-1 mRNA expression in rat epitrochlearis muscle. Biochem Biophys Res Commun. 2002;296(2):350-4

35. Hojlund K, Mustard KJ, Staehr P, Hardie DG, Beck-Nielsen H, Richter EA. AMPK activity and isoform protein expression are similar in muscle of obese subjects with and without type 2 diabetes. Am J Physiol Endocrinol Metab. 2004;286(2):E239-44.

36. Koistinen HA, Galuska D, Chibalin AV, Yang J, Zierath JR, Holman GD, et al. 5-amino-imidazole carboxamide riboside increases glucose transport and cell-surface GLUT4 content in skeletal muscle from subjects with type 2 diabetes. Diabetes. 2003;52(5):1066-72.

37. Musi N, Fujii N, Hirshman MF, Ekberg I, Fröberg S, Ljungqvist O, et al. AMP-activated protein kinase (AMPK) is activated in muscle of subjects with type 2 diabetes during exercise. Diabetes. 2001;50(5):921-7.

38. Sriwijitkamol A, Ivy JL, Christ-Roberts C, DeFronzo RA, Mandarino LJ, Musi N. LKB1-AMPK signaling in muscle from obese insulin-resistant Zucker rats and effects of training. Am J Physiol Endocrinol Metab. 2006;290(5):E925-32

39. Zheng D, MacLean PS, Pohnert SC, Knight JB, Olson AL, Winder WW, et al. Regulation of muscle GLUT-4 transcription by AMP-activated protein kinase. J Appl Physiol. 2001;91(3):1073-83.

40. Holmes BF, Sparling DP, Olson AL, Winder WW, Dohm GL. Regulation of muscle GLUT4 enhancer factor and myocyte enhancer factor 2 by AMP-activated protein kinase. Am J Physiol Endocrinol Metab. 2005;289(6):E1071-6 